\title{
Analysis of Response to the Wild Hornet Crisis Based on the SIR Model
}

\author{
Aihua Gu $(D$, Mengmeng Li, Yue Ran, Zhenzhuo Wang, Shujun Li, Qifeng Xun, \\ and Jian Dong
}

School of Information Engineering, Yancheng Teachers University, Yancheng 224002, China

Correspondence should be addressed to Aihua Gu; guaihua1978@163.com

Received 25 May 2021; Revised 29 June 2021; Accepted 5 July 2021; Published 13 July 2021

Academic Editor: Jude Hemanth

Copyright (C) 2021 Aihua Gu et al. This is an open access article distributed under the Creative Commons Attribution License, which permits unrestricted use, distribution, and reproduction in any medium, provided the original work is properly cited.

\begin{abstract}
In this paper, we propose 4 theoretical models to deal with the wild hornet crisis. First, we use ORIGIN to visualize the distribution of wild wasps. Using the least square method and the grey system GM $(1,1)$, we establish a theoretical model to predict the propagation of wild wasps over time and analyze the accuracy of the model. However, the accuracy of our model is not very high, which results from the influence of many factors such as climate and human. Secondly, we use convolution neural network to recognize the images. With the increase of network depth, the accuracy rate reaches a bottleneck, which can help predict mistaken classification. We also use the SIR infectious disease model based on the dataset file provided. In the model, we mark the confirmed giant hornet as the infected state $I$ (infected), mark the nonwild wasp as the removed state $R$ (removed, refractory, or recovered), and mark the unclassified and unverified wild wasp as the susceptible state $S$ (susceptible). A model to predict the possibility of misclassification was established by considering the normal death of wild wasp. Thirdly, by analogy with the SIR model, when the epidemic occurs, people pay more attention to the infected person. Thus, the SIR model will lead to the most likely positive sightings. Then, in order to ensure the timeliness and accuracy, the model must be updated once a year by changing or adding parameter according to local conditions. Finally, by establishing an optimized SIR infectious disease model, we added the factor of the Washington state's control of wild wasps. The analysis shows that the number of infected $I$ (i.e., wild wasps) has tended to zero after 250 days, so it can be proved that the Washington state has eliminated the pest.
\end{abstract}

\section{Introduction}

1.1. Background. In September 2019, a group of wild wasps (also known as the Asian Bumblebee) were found in Vancouver Island, British Columbia, and Canada and there have been several sightings of the pest in neighboring Washington state and many not a bumblebee.

The wasp has a similar lifetime to many other wasps. The fertilized queen appears in the spring and begins a new group. In autumn, the new queen leaves the nest and spends winter in the soil waiting for spring. A new queen nests in a range of $30 \mathrm{~km}$.

The presence of wild bees causes great anxiety due to a potentially serious impact on local bees. Washington state has set up hotlines and websites for people to witness the wasps. Under these reports from the public, the state must decide how to prioritize its limited resources for further follow-up investigations.

Recently, some scholars put forward the latest mathematical epidemiological model. The new extended concept of the vaccination game, in which the mathematical epidemiology describing the physics of diffusion is merged with the evolutionary game theory to model the human decisionmaking process, was introduced by Fujimoto et al. [1]. Ito demonstrated input-to-state stability (ISS) of the SIR model of infectious diseases with respect to the disease-free equilibrium and the endemic equilibrium [2]. Functions of Lyapunov were constructed to verify that both equilibria are individually robust with respect to perturbation which determines the eventual state of populations in epidemics. Infection model of SIR was examined in the current research to propose an analytical approach for providing an explicit 
formula associated with a straightforward computation of peak time of outbreak [3]. The proposed Lyapunov functions have strictly negative derivative to not only establish ISS but also get rid of the use of LaSalle's invariance principle and popular simplifying assumptions.

For the above problems, a simulation method of wild wasp crisis is proposed based on SIR. Experimental simulation proves that the proposed method has high measurement accuracy and greatly improves the accuracy of predicting the number of wild wasps.

1.2. Our Works. We need to predict the number changes of wild wasps and their accuracy, analyze whether sightings are wild wasps, solve how to preferentially investigate the most likely sightings of wild wasps and how to prove that Washington state has eliminated the pest, and take appropriate measures to improve the current situation. We mainly focus on the following tasks.

For the above phenomena, we propose different models to analyze the propagation of wild wasps and use the obtained data to find out a method to eliminate wild wasps. Firstly, we use ORIGIN to visualize the distribution of wild wasps. Then, we establish a model to predict the propagation of wild wasps over time based on least square method and grey system GM $(1,1)$. Finally, we analyze the accuracy of our model. Because of the influence of other factors, such as climate and human factors, the accuracy is very low.

Next, we use convolution neural network to recognize the image according to the provided image file. With the increase of network depth, the accuracy rate reaches a bottleneck, which can help to classify predicting errors.

We also use the SIR infectious disease model based on the dataset file provided and regard the identified wild wasps as infected state $I(I)$, excluded as removed state $R$ ( $R$ as susceptible state $(S)$ ), a model to predict the possibility of misclassification was established by considering the normal death of wild wasp.

Finally, by establishing an optimized SIR infectious disease model, we add the element of the Washington state's control of wild wasps. The result shows that the number of infected $I$ (i.e., wild wasps) has tended to zero after 250 days, so it can be proved that the Washington state has eliminated the pest.

\section{Prediction of the Spread of Wild Wasps over Time}

Wild hornets are the world's largest wasp species, and the emergence of nests is worrying. In addition, bumblebees are predators of European bees. A small number of bumblebees can destroy the entire European bee colony in a short time. At the same time, they are greedy predators of other insects which are considered to be agricultural pests. Therefore, we need to focus on the distribution and prediction of wild wasp according to the given data. Therefore, we use least squares, grey systems, convolutional neural networks, and SIR infectious disease models to focus on the distribution and prediction of wild wasps based on the given data.
We hypothesized that all wasps except those excluded were bumblebees; that is to say, all wasps that have not been classified and verified are considered to be bumblebees. In this way, we can increase the number of samples which is helpful for our research $[4,5]$.

According to the previous wild wasp data, by using ORIGIN by years, we map the geographical distribution of wild wasps, in which the transverse coordinate represents the longitude and the vertical coordinate represents the latitude, as shown in Figures 1-12.

According to the geographical distribution map of wild wasps in each year, we can presumably speculate that the number of wild wasps shows an overall upward trend, and in recent years, growth rate has increased significantly. The diffusion law is to show a star shape spreading around. To further study the diffusion law of wild wasps, we also need to establish the least squares model and the grey system model.

2.1. Predicting the Propagation of Wild Hornets by LSE. Least square method (LSE) [6] is a mathematical optimization technique. It seeks the best function match of the data by minimizing the sum of squares of errors. Using the least square method, the unknown data can be easily obtained, and the sum of the squares of the error between the obtained data and the actual data can be minimized. Least square method can also be used for curve fitting. Some other optimization problems can also be expressed by minimizing energy or maximizing entropy by the least square method.

2.1.1. Establishing the Least Square Model. The following is the number of wild wasps found in 2019 and 2020, and we will use the 2019 data to predict the 2020 data and then compare the 2020 data with the 2020 real data to verify the feasibility of the model as shown in Tables 1 and 2 .

Regression model is a mathematical model for quantitative description of statistical relationships. For example, the mathematical model of multiple linear regression can be expressed as $y=\beta_{0}+\beta_{1} * x+\varepsilon_{i}$, where $\beta_{0}, \beta_{1}, \ldots \beta_{p}$ is $p+1$ parameter to be estimated, $\varepsilon_{i}$ is independent of each other and subject to the same normal distribution $N\left(0, \sigma^{2}\right)$ random variables, and $y$ is a random variable. In the equation, $x$ can be a random number or a nonrandom number, and $\beta i$ is a regression coefficient which is used to characterize the degree of influence of independent variables on dependent variables.

Let the regression linear equation be $Y=A+B X$ :

$$
\begin{aligned}
& B=\frac{\sum(X-\bar{X})(Y-\bar{Y})}{\sum(X-\bar{X})^{2}}, \\
& A=\bar{Y}-B \bar{X} .
\end{aligned}
$$

Using data to obtain parameters, $A=138.84848$ and $B=7.04895$. So, the regression equation is $Y=138.84848+7.04895 X$. 


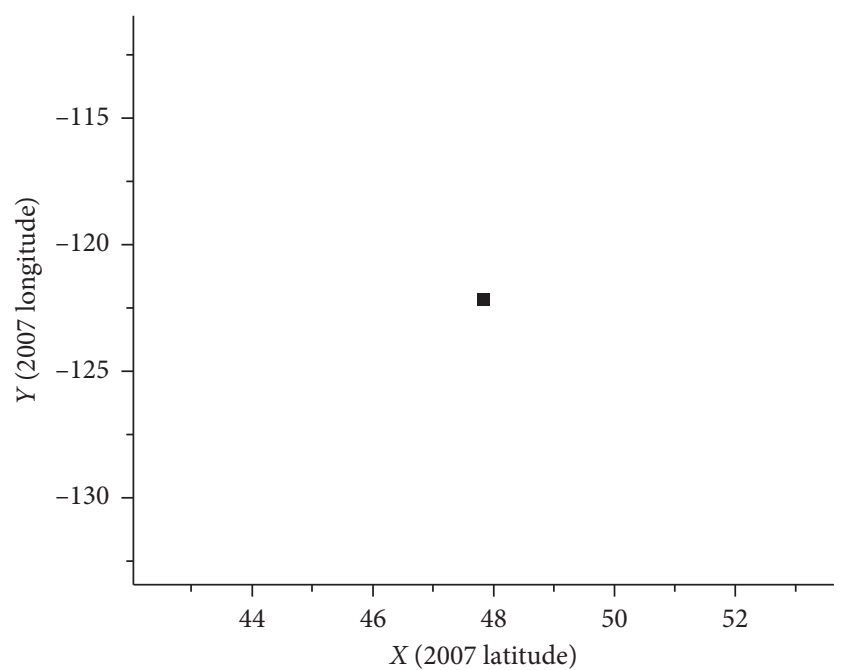

FIgURe 1: 2007 distribution map.

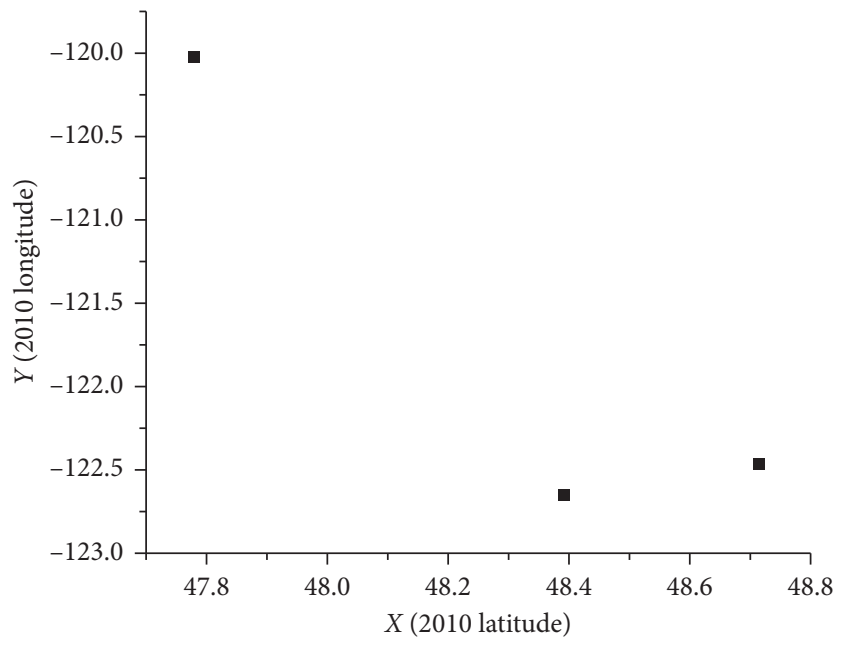

Figure 2: 2010 distribution map.

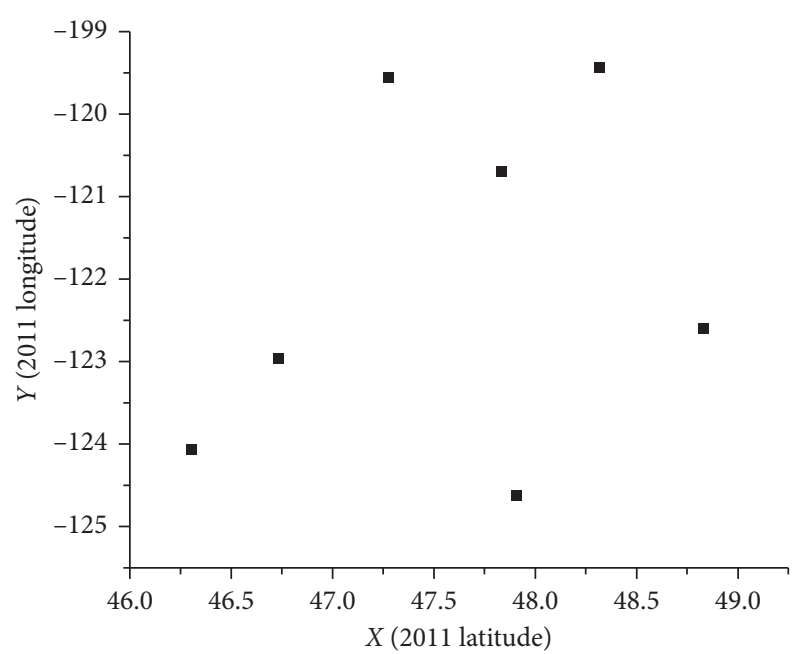

Figure 3: 2011 distribution map.

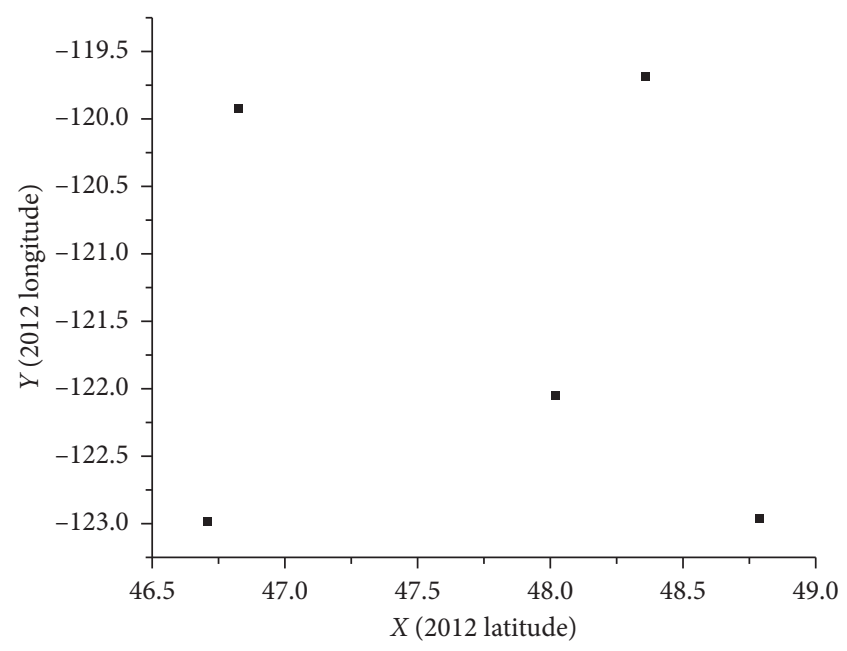

Figure 4: 2012 distribution map.

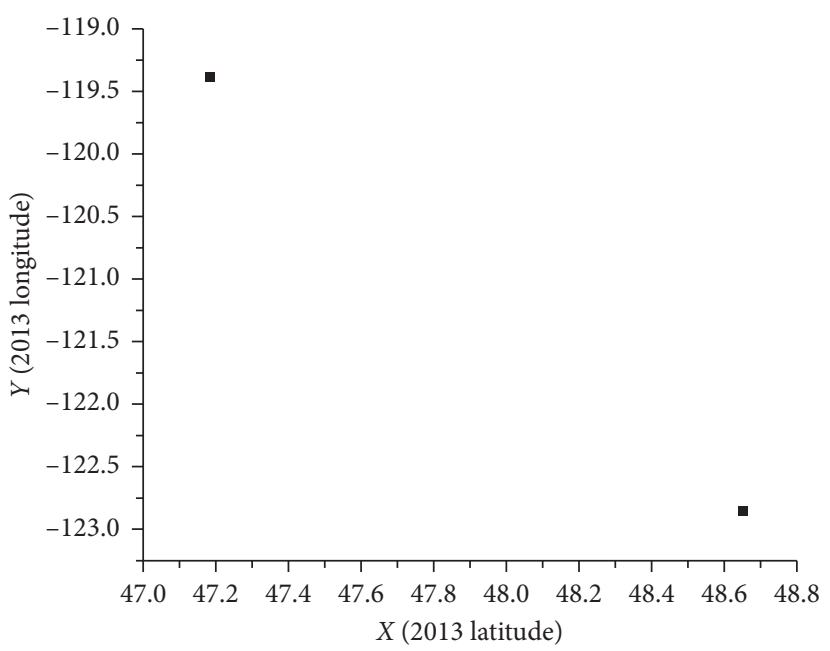

Figure 5: 2013 distribution map.

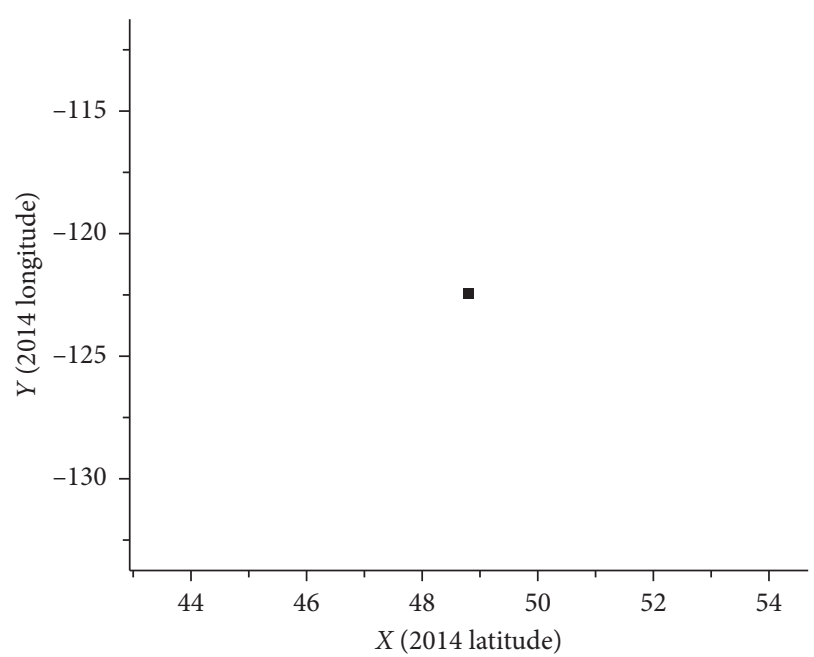

FiguRe 6: 2014 distribution map. 


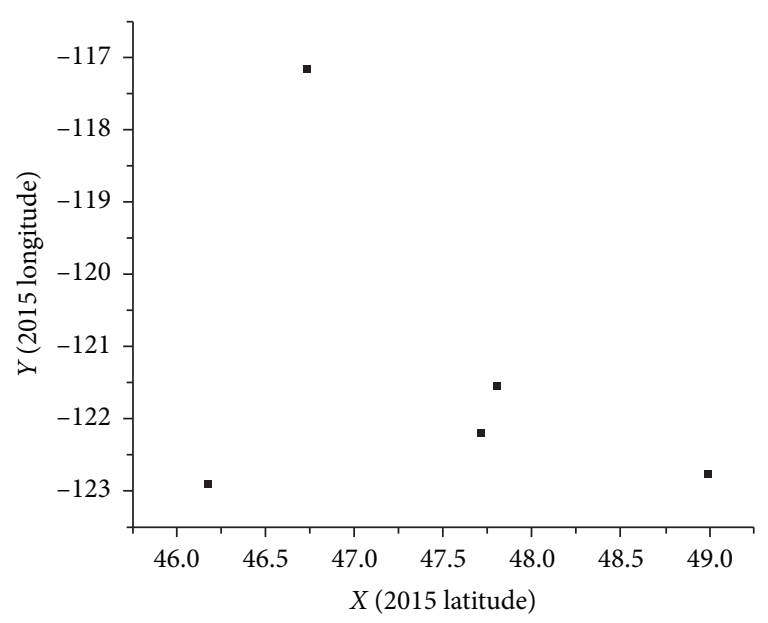

Figure 7: 2015 distribution map.

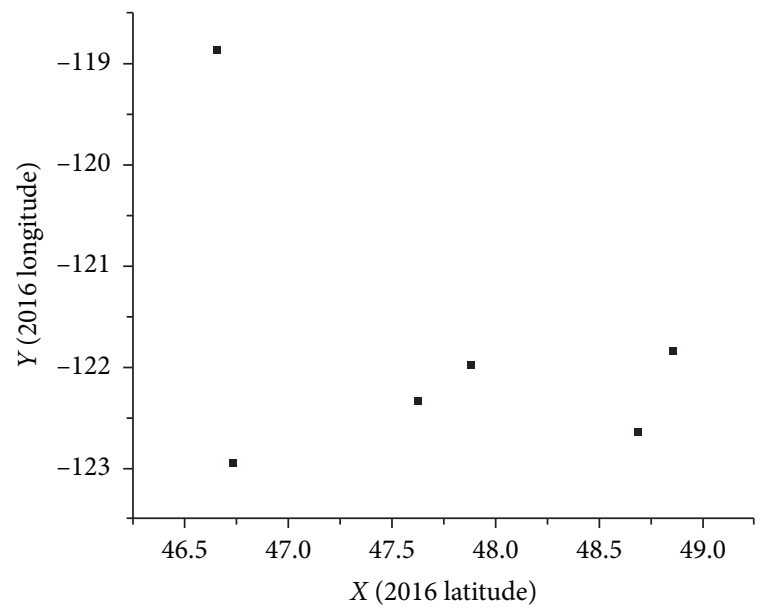

Figure 8: 2016 distribution map.

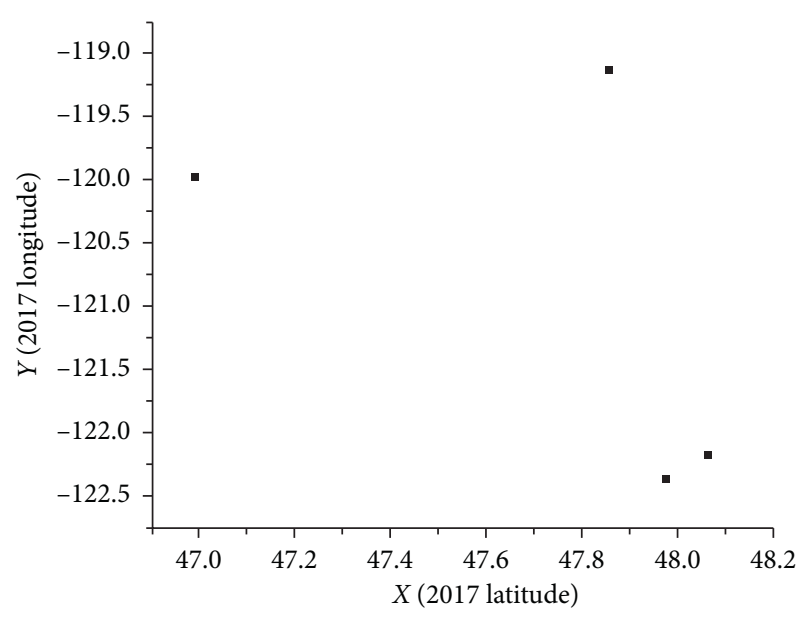

Figure 9: 2017 distribution map.

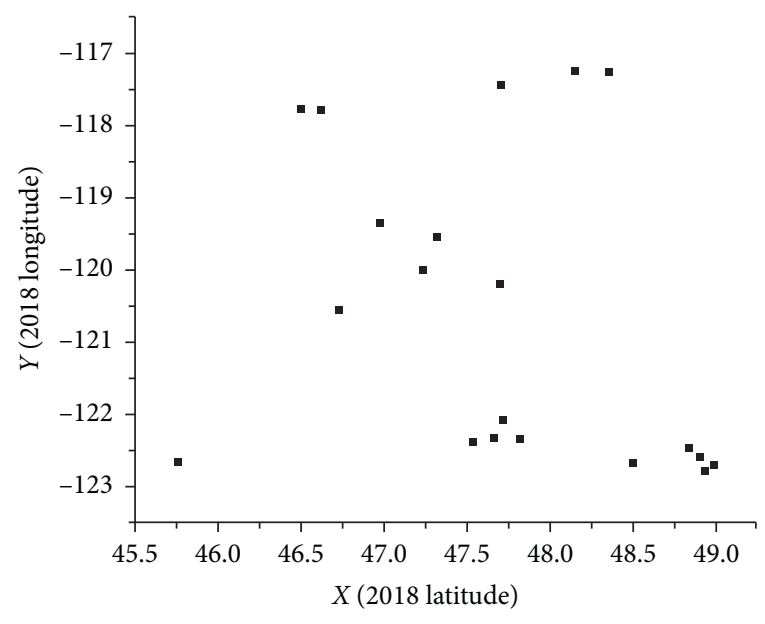

Figure 10: 2018 distribution map.

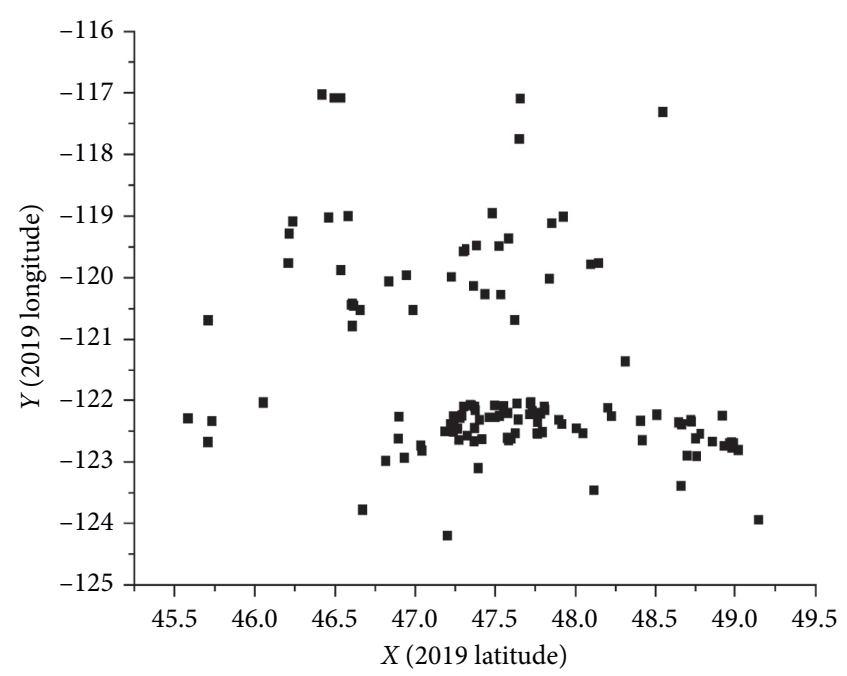

FIgURE 11: 2019 distribution map.

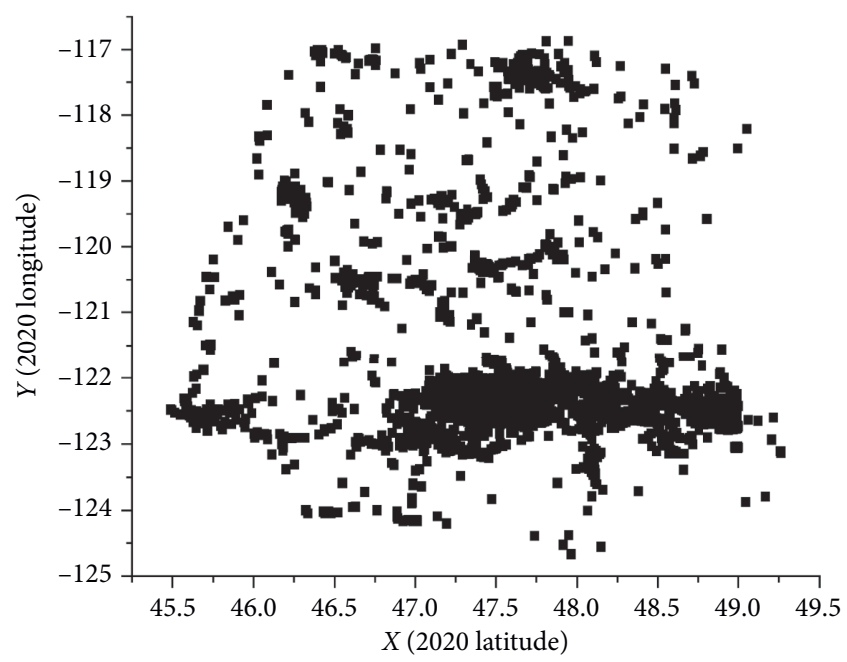

Figure 12: 2020 distribution map. 
TABLE 1: Number of bumblebees found in 2019.

\begin{tabular}{lcccccccccccc}
\hline Month & 1 & 2 & 3 & 4 & 5 & 6 & 7 & 8 & 9 & 10 & 11 & 12 \\
\hline Number $(s)$ & 1 & 0 & 1 & 1 & 8 & 12 & 24 & 31 & 17 & 7 & 3 & 2 \\
\hline
\end{tabular}

TABLE 2: Number of bumblebees found in 2020.

\begin{tabular}{lcccccccccccc} 
Month & 1 & 2 & 3 & 4 & 5 & 6 & 7 & 8 & 9 & 10 & 11 & 12 \\
\hline Number $(s)$ & 1 & 2 & 17 & 146 & 441 & 134 & 448 & 640 & 286 & 89 & 1 & 1
\end{tabular}

2.1.2. Least Square Model Test. In this paper, we used $t$-test to detect the significance of regression coefficient.

Residual standard deviation is

$$
S y=\sqrt{\sum \frac{(Y-\bar{Y})^{2}}{n-2}} .
$$

Standard error of regression coefficient is

$$
S b=\frac{S y}{\sqrt{\sum(X-\bar{X})^{2}}} .
$$

According to the data of bumblebee in 2019, the data of bumblebee in 2020 are predicted by the model, and the following error chart is made. The red line represents the data predicted by the least square method, and the black point represents the actual data at that time as shown in Figure 13.

It can be observed from the diagram that the propagation of wild hornet in 2020 predicted by the least square method has a certain deviation compared with the actual results, but the average value of the actual data in this year is the same as the average value of the predicted data. Thus, the least square method is effective.

\subsection{Predicting Wild Hornet Propagation in the Coming December by the Grey System Model}

2.2.1. Modelling GM (1, 1) Grey System. To establish grey prediction model [7], we assume that $x_{(0)}=\left\{x_{(0)}(k)\right.$, $k=1,2, \ldots, n\}$ is a nonnegative monotone original data column of a prediction object.

First, a cumulative sequence is generated by accumulating $X_{(0)}$ :

$$
\begin{gathered}
x_{(1)}=\left\{x_{(1)}(k), k=1,2, \ldots, n\right\}, \\
x_{(1)}(k)=\sum_{i=1}^{k} i=x_{(1)}(k-1)+x_{(0)}(k) .
\end{gathered}
$$

$\operatorname{GM}(1,1)$ is $\left(\mathrm{d} x_{(1)} / \mathrm{d} t\right)+a x_{(1)}=u$.

Let the parameter sequence be $\widehat{a}$ and $\widehat{a}=[a, u]^{T}$. $\widehat{a}$ is derived by solving the following formula: $\widehat{a}=\left(B^{T} B\right)^{-1} B^{T} Y_{n}$, where $B$ is computed by the following equation:

$$
\begin{gathered}
B=\left[\begin{array}{cc}
-\frac{1}{2} *\left(x_{(1)}(1)+x_{(1)}(2)\right) & 1 \\
-\frac{1}{2} *\left(x_{(1)}(2)+x_{(1)}(3)\right) & 1 \\
1 \\
-\frac{1}{2} *\left(x_{(1)}(n-1)+x_{(1)}(n)\right) & 1
\end{array}\right], \\
Y_{n}=\left(X^{(0)}(2), X^{(0)}(3), \ldots, X^{(0)}(n)\right)^{T} .
\end{gathered}
$$

Since the GM obtains a cumulative amount, the GM data must be generated inversely to use the predicted value:

$$
\begin{aligned}
\widehat{X}^{(1)}(k) & =\sum_{i=1}^{k} \widehat{X}^{(0)}(i)=\sum_{i=1}^{k-1} \widehat{X}^{(0)}(i)+\widehat{X}^{(0)}(k), \\
\widehat{X}^{(0)}(k) & =\widehat{X}^{(1)}(k)-\sum_{i=1}^{k-1} \widehat{X}^{(0)}(i), \\
\widehat{X}^{(1)}(k-1) & =\sum_{i=1}^{k-1} \widehat{X}^{(0)}(i), \\
\widehat{X}^{(0)}(k) & =\widehat{X}^{(1)}(k)-\widehat{X}^{(1)}(k-1) .
\end{aligned}
$$

2.2.2. Testing of Grey System Models. Set the original sequence as

$$
X_{(0)}=\left\{x_{(0)}(1), x_{(0)}(2), \ldots, x_{(0)}(n)\right\} .
$$

The corresponding model simulation sequence is

$$
\widehat{X}_{(0)}=\left\{\widehat{x}_{(0)}(1), \widehat{x}_{(0)}(2), \ldots, \widehat{x}_{(0)}(n)\right\} .
$$

The residual sequence is

$$
\begin{aligned}
\mathcal{E}_{(0)} & =\left\{\varepsilon_{(1)}, \varepsilon(2), \ldots, \varepsilon(n)\right\} \\
& =\left\{x_{(0)}(1)-\widehat{x}_{(0)}(1), x_{(0)}(2)-\widehat{x}_{(0)}(2), \ldots, x_{(0)}(n)-\widehat{x}_{(0)}(n)\right\} .
\end{aligned}
$$




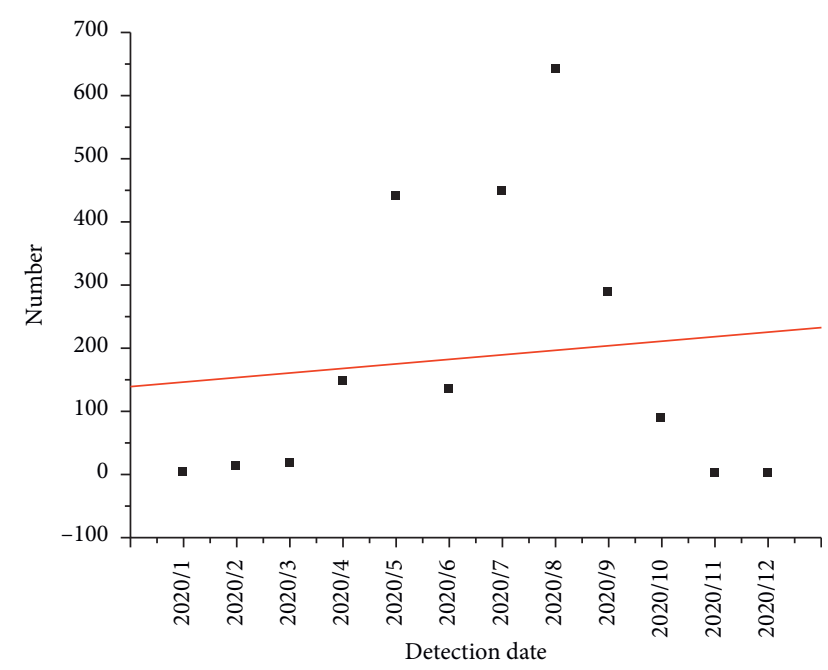

Figure 13: Variegated wild bees over time.

The relative error sequence is

$$
\Delta=\left\{\left|\frac{\varepsilon(1)}{x_{(0)}(1)}\right|,\left|\frac{\varepsilon(2)}{x_{(0)}(2)}\right|, \ldots,\left|\frac{\varepsilon(n)}{x_{(0)}(n)}\right|\right\}=\left\{\Delta_{k}\right\}_{1}^{n} .
$$

For $k<n, \Delta k=\left|\varepsilon(k) / x_{(0)}(k)\right|$ is $k$ relative error of point simulation, $\Delta_{(n)}=\left|\varepsilon(n) / x_{(0)}(n)\right|$ is defined as the relative error of filtering, $\bar{\Delta}=(1 / n) \sum_{k=1}^{n} \Delta_{k}$ is called the mean simulation relative error, and $1-\Delta$ means relative accuracy, and $1-\Delta_{n}$ is called the filtering accuracy.

Given $\alpha$, if $\bar{\Delta}<\alpha$ and $\Delta_{n}<\alpha$, the model is called residual qualified model.

We establish a grey system prediction model GM $(1,1)$ using local 2019 wild wasp breeding data, and the monthly propagation of wild wasp in 2020 is predicted. We compared the predicted values with the actual data. We use ORIGIN to draw a point map by taking the month as the $x$-axis and the number of wasps as the $y$-axis, as shown in Figure 14. The red dot represents the forecast data, and the blue point represents the real data of the month.

2.3. Precision Analysis. Using the above least square method and grey system algorithm, the number of wild wasps found in the next 16 days and 12 months was predicted, respectively. The results are shown in Table 3.

Due to the influence of climate, human, and other factors, the accuracy is low.

In addition, if the annual number of bumblebees varies from high to low, it is greatly affected by unnatural factors, and the data is not referential. Therefore, the annual prediction will not be discussed for the time being.

\section{Image Recognition of Wild Hornet Based on Convolutional Neural Network}

The emergence of wild wasps caused panic among the masses, so the masses uploaded a lot of data on wild wasps, among which a lot are mistaken cases. In order to save

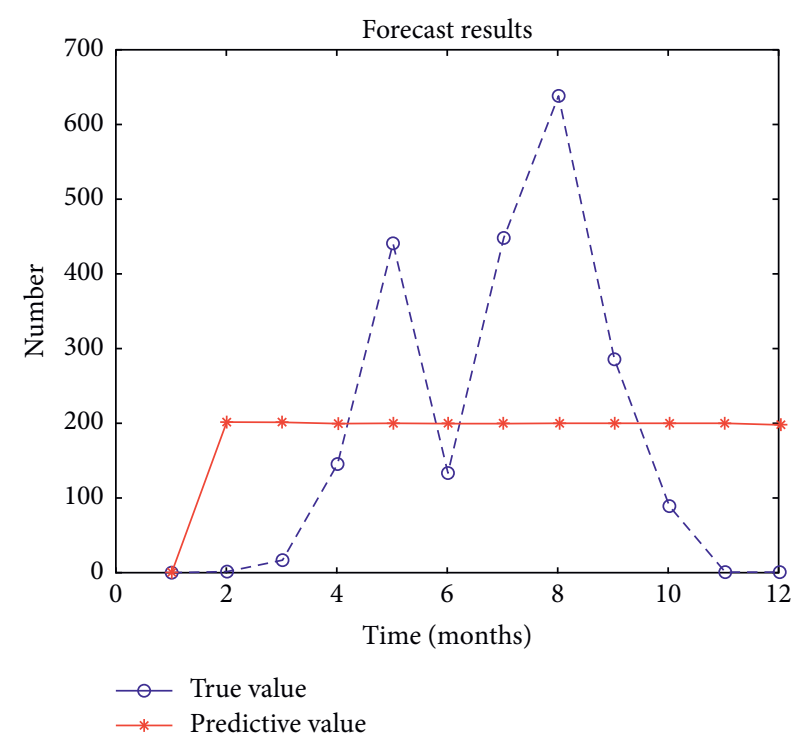

FIgURE 14: Comparison of true and predicted values.

government resources and locate wild wasps, we built a model through convolution neural network which can help to automatically recognize the uploaded image files and accurately identify the wild hornet.

Due to the large number of given image materials, in order to ensure that the algorithm learns useful data, we have roughly sorted out the materials, including deleting too vague pictures and selecting representative pictures in video files for screenshots.

3.1. Introduction to Convolutional Neural Networks. Convolution neural network $[8,9]$ contains a feature extractor composed of convolution layer and subsampling layer. In the convolution layer of convolution neural network, one neuron is only connected with some adjacent layer neurons. A convolution layer in a CNN usually contains several feature planes, each of which is composed of some rectangular arranged neurons, and the neurons in the same feature plane share weights, where the shared weights are convolution kernels. Convolution kernel is usually initialized in the form of random decimal matrix. In the training process of network, convolution kernel will learn reasonable weights. Subsampling usually has two forms: mean subsampling and maximum subsampling. Subsampling can be regarded as a special convolution process. Convolution and subsampling greatly simplify the model complexity and reduce the parameters of the model. The basic data of the convolution kernel are shown in Table 4.

3.2. Reverse Algorithm Model of Convolutional Neural Network. Backpropagation algorithm is the most commonly used algorithm with minimum error for training convolutional neural network. The main principles are as follows: the DataTrain data is input into the convolution layer of the $\mathrm{CNN}$, then it passes through the neuron, and finally it reaches the convolution kernel and outputs the result. The 
TABle 3: Forecast results.

\begin{tabular}{lccr}
\hline \multicolumn{2}{c}{$2021 / 1 / 1-2021 / 1 / 16$} & & $2021 / 1-2021 / 12$ \\
Day & Number (number) & Month & Number (number) \\
\hline $2021 / 1 / 1$ & 230 & $2021 / 1$ & 205 \\
$2021 / 1 / 2$ & 237 & $2021 / 2$ & 207 \\
$2021 / 1 / 3$ & 244 & $2021 / 3$ & 209 \\
$2021 / 1 / 4$ & 251 & $2021 / 4$ & 211 \\
$2021 / 1 / 5$ & 258 & $2021 / 5$ & 213 \\
$2021 / 1 / 6$ & 265 & $2021 / 6$ & 215 \\
$2021 / 1 / 7$ & 272 & 20217 & 217 \\
$2021 / 1 / 8$ & 279 & $2021 / 8$ & 219 \\
$2021 / 1 / 9$ & 286 & $2021 / 9$ & 221 \\
$2021 / 1 / 10$ & 293 & $2021 / 10$ & 223 \\
$2021 / 1 / 11$ & 300 & $2021 / 11$ & 225 \\
$2021 / 1 / 12$ & 307 & & 227 \\
$2021 / 1 / 13$ & 314 & & \\
$2021 / 1 / 14$ & 321 & & \\
$2021 / 1 / 15$ & 330 & & \\
$2021 / 1 / 16$ & 337 & & \\
\hline
\end{tabular}

TABLE 4: Convolution core data table.

\begin{tabular}{lccc}
\hline Type of operation & Convolution nucleus/step length & Output dimension & Number of parameters \\
\hline Input & - & $227 \times 227 \times 3$ & - \\
Conv1/BN & $11 \times 11 / 4$ & $55 \times 55 \times 96$ & 34848 \\
MaxPool1 & $3 \times 3 / 2$ & $27 \times 27 \times 96$ & - \\
Conv2/BN & $5 \times 5 / 2$ & $14 \times 14 \times 256$ & 614400 \\
MaxPool2 & $3 \times 3 / 2$ & $6 \times 6 \times 256$ & - \\
Conv3/BN & $3 \times 3 / 1$ & $6 \times 6 \times 384$ & 884736 \\
Conv4/BN & $3 \times 3 / 1$ & $6 \times 6 \times 384$ & 1327104 \\
Conv5/BN & $3 \times 3 / 1$ & $6 \times 6 \times 256$ & 884736 \\
MaxPool3 & $3 \times 3 / 2$ & $3 \times 3 \times 256$ & - \\
Conv6 & $1 \times 1 / 1$ & $3 \times 3 \times 2048$ & 524228 \\
Conv7 & $1 \times 1 / 1$ & $3 \times 3 \times 2048$ & 4194304 \\
GAP & $3 \times 3 / 1$ & $1 \times 1 \times 2048$ & - \\
Output & - & 8 & 16384 \\
\hline
\end{tabular}

error between the output result and the real value is calculated, and the error is backpropagated from the convolution core to the neuron until the convolution layer of CNN. The above process is iterated until convergence. Schematic diagram of the convolutional network structure is shown in Figure 15.

The calculation process of neural network takes the 3layer structure as an example and the parameters of neural network are $(W, b)=\left(W^{(1)}, b^{(1)}, W^{(2)}, b^{(2)}\right)$, where $W_{i j}^{(l)}$ is the weight parameter between the $j$ neuron of the $l$ layer and the $i$ neuron of the first layer, $b_{i}^{(l+1)}$ is the bias term for the $i$ neuron on layer $l+1, W^{(1)} \in R^{3 \times 3}, W^{(2)} \in R^{1 \times 3}$, the total output of the bias term is 1 , and $a_{i}^{(1)}$ represents the output value of the $i$ neuron in layer $l$. At $l=1, a_{i}^{(1)}=x_{i}$.

The formulas for calculating neural networks are as follows:

$$
\begin{aligned}
& a_{1}^{(2)}=f\left(W_{11}^{(1)} x_{1}+W_{12}^{(1)} x_{2}+W_{13}^{(1)} x_{3}+b_{1}^{(1)}\right), \\
& a_{2}^{(2)}=f\left(W_{21}^{(1)} x_{1}+W_{22}^{(1)} x_{2}+W_{23}^{(1)} x_{3}+b_{2}^{(1)}\right), \\
& a_{3}^{(2)}=f\left(W_{31}^{(1)} x_{1}+W_{32}^{(1)} x_{2}+W_{33}^{(1)} x_{3}+b_{3}^{(1)}\right) .
\end{aligned}
$$

Calculate the output with $h_{w}, b^{(x)}$ :

$$
h w, b^{(x)}=a_{1}^{(3)}=f\left(W_{11}^{(2)} a_{1}^{(2)}+W_{12}^{(2)} a_{2}^{(2)}+W_{13}^{(1)} a_{3}^{(2)}+b_{1}^{(1)}\right) .
$$

Given a set of $m$ samples $M=\left\{\left(x^{(1)}, y^{(1)}\right)\right.$, $\left.\left(x^{(2)}, y^{(2)}\right), \ldots,\left(x^{(m)}, y^{(m)}\right)\right\}$, then the whole loss function of neural network can be expressed as

$$
J(W, b)=\left[\frac{1}{m} \sum_{i=1}^{m}\left(\frac{1}{2\left|h_{w}, b\left(x^{(i)}\right)-y^{(i)}\right|^{2}}\right)\right]+\frac{\lambda}{2} \sum_{l=1}^{n_{1}-1} \sum_{i=1}^{S_{l}} \sum_{j=1}^{S_{l}+1}\left(W_{j i}^{(1)}\right)^{2} .
$$




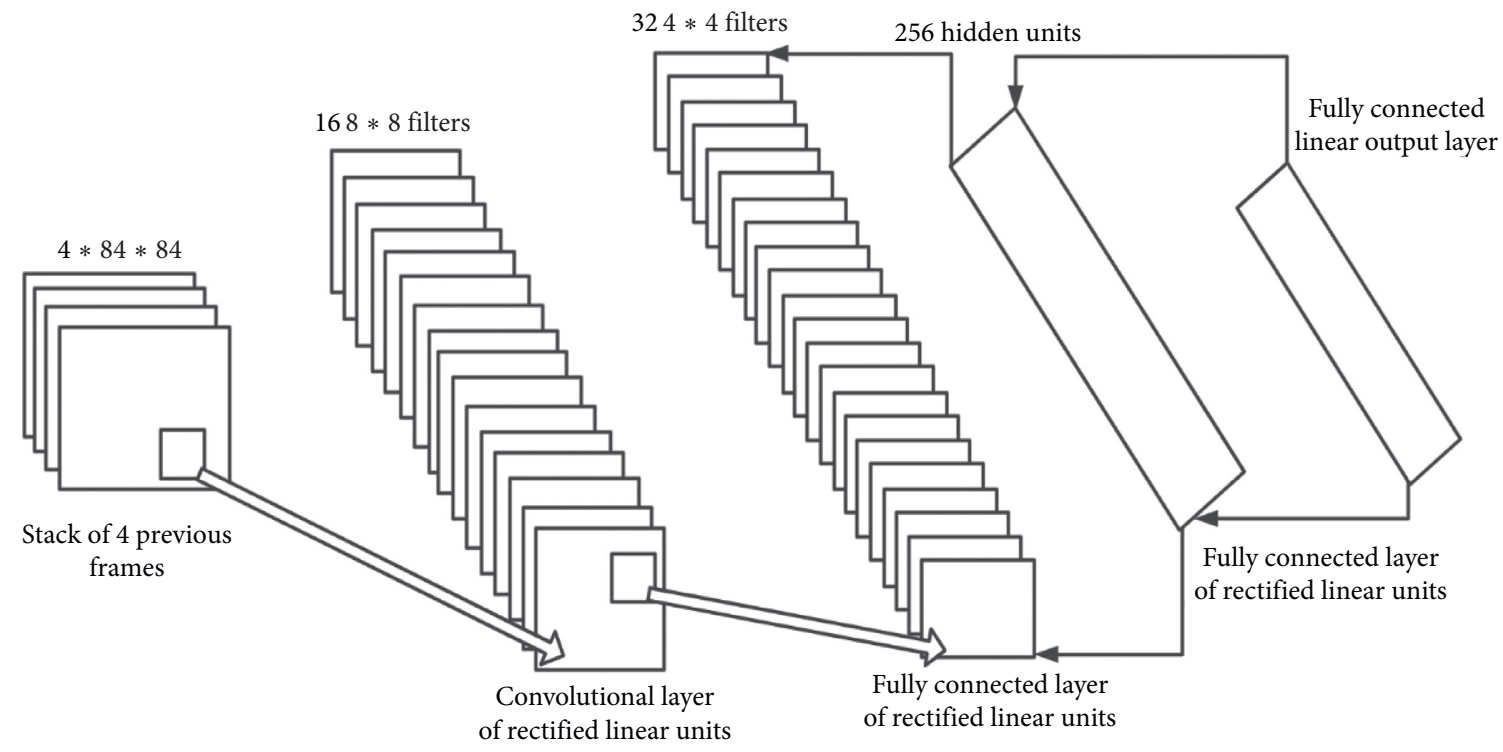

Figure 15: Schematic illustration of the convolutional network structure.

Here, $W$ and $b$ represent weight parameters and bias terms, respectively, and $\lambda$ is the weight attenuation factor. In this paper, we used stochastic gradient descent algorithm to update the weights and bias terms in the network, and the formula is as follows:

$$
\begin{aligned}
& W_{i j}^{(1)}=W_{i j}^{(1)}-\alpha \frac{\vartheta}{\vartheta W_{i j}^{(1)}} J(W, b), \\
& b_{i}^{(l+1)}=b_{i}^{(1)}-\alpha \frac{\vartheta}{\vartheta b_{i}^{(l+1)}} J(W, b) .
\end{aligned}
$$

Here, $\alpha$ is the learning rate.

The two formulas represent the partial derivative of the loss function to the network weight and the bias term, respectively.

Using convolution neural network model to recognize the images [10-14], most of the wasps can be identified for the next data analysis. Although there is a certain deviation, it does not affect the overall results.

\section{Analysis of Wild Hornet Data Based on the Infectious Disease Model}

The status of $\mathrm{N}$ individuals within a population (population) in a typical infectious disease model can be divided into the following categories:

(1) The first category is $S$ susceptible, that is, healthy but susceptible individuals.

(2) The second is the infected state $I$ ( $I$ affected), where the infected individuals can also infect the healthy individuals.

(3) The third is removed state $R$ ( $R$ modified, $R$ fragile, or compromised), also known as immune state and recovery state. After an individual has gone through a complete infection cycle, the individual is no longer infected, so the individual can no longer be considered.

4.1. Establishment of an Infectious Disease Model. In this problem, we can regard the confirmed wasps as infected state $I$ (infected), those that have been excluded as $R$ (removed, refractory, or recovered), and those that have not been classified or verified as susceptible state $S$ (susceptible).

Based on the above three assumptions, the infection model is as follows:

$$
\left\{S(i)+I(j) \longrightarrow^{\beta} I(i)+I(j), I(i) \longrightarrow^{\gamma} R(i) .\right.
$$

Based on the above three basic assumptions, we can see that when the susceptible individual and the infected individual are fully mixed, the growth rate of the infected individual is $\beta i(t) s(t)-\gamma i(t)$, the decline rate of susceptible individuals was $\beta i(t) s(t)$, and the growth rate of the restored individual is $\gamma i(t)$. The process of susceptibility from disease to migration can be expressed by differential equations as follows:

$$
\left\{\begin{array}{l}
\frac{\mathrm{d} s(t)}{\mathrm{d} t}=-\beta i(t) s(t), \\
\frac{\mathrm{d} i(t)}{\mathrm{d} t}=\beta i(t) s(t)-\gamma i(t), \\
\frac{\mathrm{d} r(t)}{\mathrm{d} t}=\gamma i(t) .
\end{array}\right.
$$

The solution of the differential equation is

$$
I=\left(S_{0}+I_{0}\right)-S+\frac{1}{\sigma} \ln \frac{S}{S_{0}},
$$

where $S_{0}$ and $I_{0}$ represent initial values, $\sigma$ was the number of contacts in infectious period, and 


$$
\alpha=\frac{\beta}{\gamma} .
$$

4.2. Solution of the Infectious Disease Model. On the basis of the above theorem, a model can be established to solve the problem. In 365 days of 2020, the confirmed wasps were regarded as infected state $I$ (infected), the excluded wasps were regarded as state $R$ (removed, refractory, or recovered), and the unclassified and unverified wasps were regarded as susceptible state $S$ (susceptible). Using the optimized SIR model, the results are shown in Figure 16, where $D$ is the number of wasps that died without human control.

According to the diagram, the SIR model based on differential equations can fit the curve accurately according to the existing data, and the measures to prevent wasps from spreading can be obtained by using phase trajectory analysis. The theoretical basis is sufficient.

In order to ensure the timeliness and accuracy of the model, it is necessary to update the model by changing the parameter value or adding the parameter according to the local situation, and the update frequency is one year.

4.3. The Spread of Infectious Disease Models and Evidence That Washington Has Eliminated Wild Wasps. Because of the potential serious impact on the local bees, the presence of wild bees can cause great anxiety. Washington has set up a hotline and website for people to report sightings of the wasps. Based on these public reports, the state must decide how to prioritize the use of its limited resources for further follow-up investigations.

Assuming that Washington has taken a series of control measures to inhibit the growth of wild wasps, taking the 2020 data of wild wasps as an example, we can add the control measures of Washington state to establish an improved SIR infectious disease model [15] to verify whether Washington has eliminated the wild wasps. Using the optimized SIR model, the results are shown in Figure 17.

Suppose the state of Washington did not take control in the first 46 days, the state of infection $I$ (i.e., confirmed as wild wasp) showed a power law upward trend. The state of Washington took control measures in the 47th day, and the number of infections $I$ (i.e., confirmed as wild wasp) after 250 days had tended to zero, so it can be proved that the state of Washington eliminated the pest.

\section{Error/Sensitivity Analysis}

5.1. Sensitivity Analysis of the Least Square Method. It can be used for curve fitting. Some other optimization problems can also be formulated by minimizing energy or maximizing entropy by the least square method. When the independent variable and the dependent variable have random error of zero mean and the same variance at the same time, this method can give the best fitting in statistical sense. Experimental results of the least squares are in Table 5.

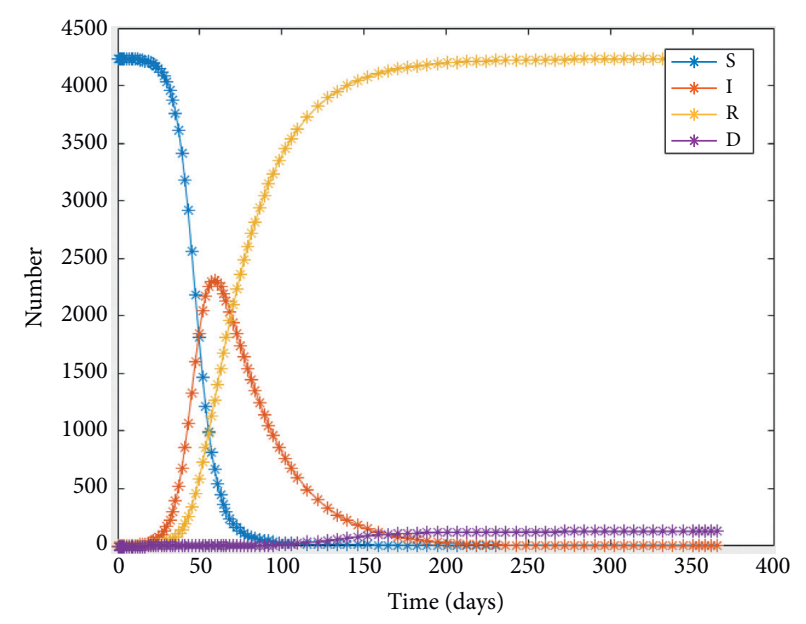

Figure 16: $S, I, R, D$ quantitative change chart.

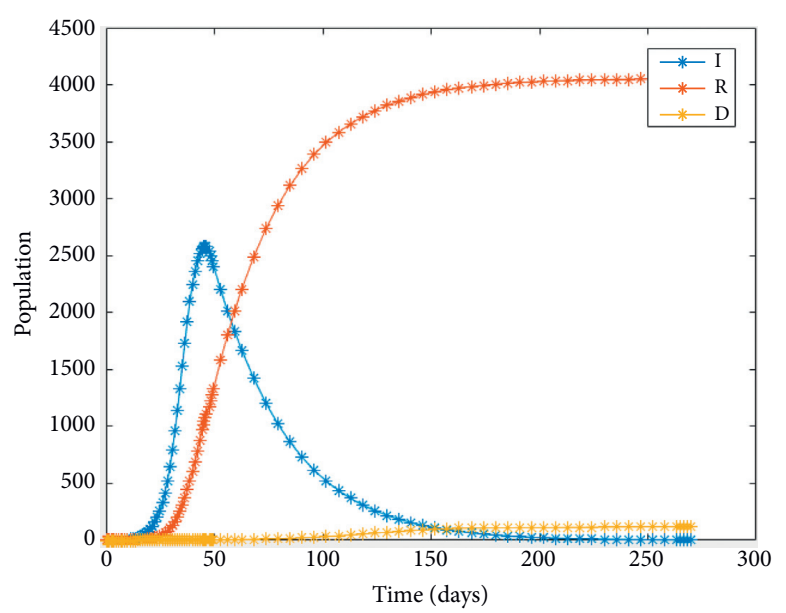

Figure 17: Comparison of government prevention and control.

But when XTX is irreversible, the least square estimation cannot be used. The least square method is a linear estimation, which is a linear relationship by default, and has some limitations. In the process of regression, the correlation of regression cannot pass through each regression data point. Therefore, the prediction error of the number of wild wasps in the future is large.

5.2. Sensitivity Analysis of the Grey Prediction Model. Grey prediction model has a good performance on complex systems with uncertain factors and requires less sample data. However, grey prediction model based on exponential rate does not consider the randomness of the system, and the medium and long-term prediction accuracy is poor.

5.3. Sensitivity Analysis of Convolutional Neural Networks. In the image recognition of wild hornet, we have established a convolution neural network model for image recognition. Because image recognition is based on layer-by-layer analysis of images, if only the number of convolution layers 
TABLE 5: Least square method test results.

\begin{tabular}{lcccc}
\hline Optimization function & Solution result & Number of iterations & Number of calls to a function & Calculating time \\
\hline Fmincon & 0.02537 & 11 & 69 & 0.261 \\
Fminsearch & 0.02535 & 66 & 116 & 0.273 \\
Lsqnonlin & 0.02535 & 2 & 12 & 0.160 \\
\hline
\end{tabular}

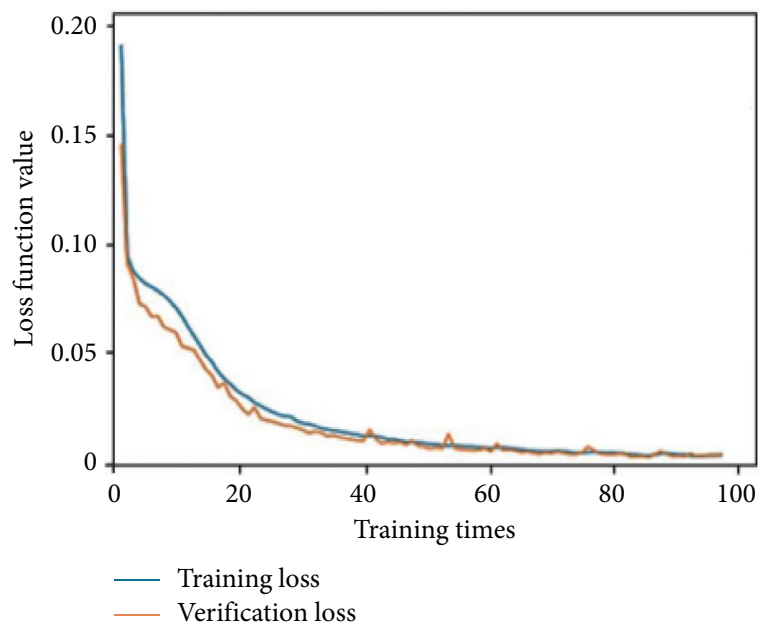

FIgURe 18: Training loss table.

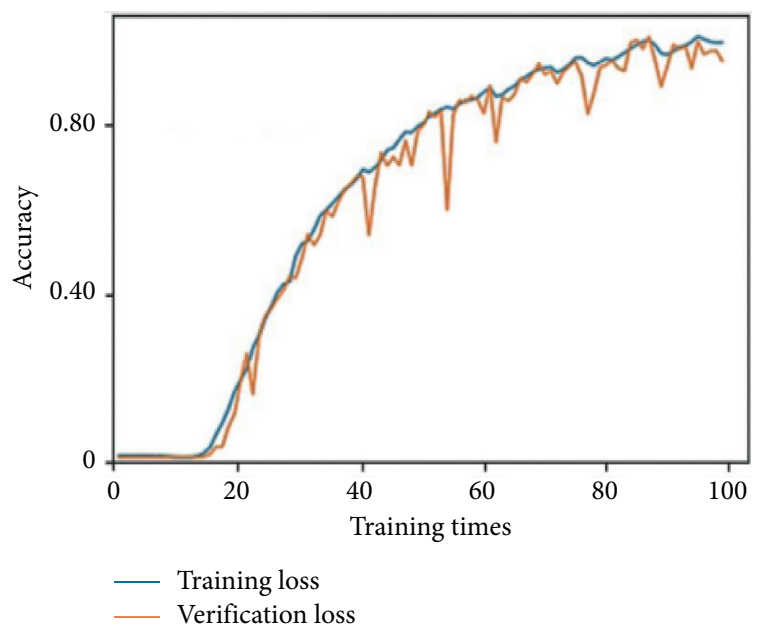

FIgURE 19: Training accuracy table.

is simply stacked, it will lead to the disappearance or explosion of gradient in the network, which fundamentally leads to the failure of function convergence. However, we can solve this problem well by using backpropagation through standard initialization and intermediate standardization layer so that the network can begin to converge the function by random gradient descent in the process of backpropagation. The general depth selection is not more than 10 layers, and we generally have three layers to study the problem of wild Hornet.
5.4. Error Analysis of Convolutional Neural Network. Convolution neural network can play a fast and effective role for the network with suitable depth. However, when the deep network begins to converge, the problem of network degradation is exposed. As the depth of the network increases, the accuracy reaches a bottleneck first, and then it begins to decrease rapidly. Notably, this degradation is not caused by overfitting. For a network with appropriate depth, increasing the number of layers will lead to an increase in the training error rate. Figure 18 is a training loss table, and Figure 19 is a training accuracy table.

In addition, the increase of the number will also lead to image training errors. Some images will lead to image recognition errors due to pixel reasons, resulting in judgment errors. For checking the recognition effect of the model, the harmonic mean value $(F)$ is used as the comprehensive evaluation index. The formula is as follows:

$$
\begin{aligned}
& P=\frac{\mathrm{RS}}{\mathrm{RS}+\mathrm{FS}}, \\
& R=\frac{\mathrm{RS}}{\mathrm{RS}+\mathrm{FN}}, \\
& F=\frac{2 P R}{P+R},
\end{aligned}
$$

where $P$ represents the correct rate, $R$ represents the recall rate, $\mathrm{RS}$ represents the correct recognition of the target in the image, FS represents the incorrect recognition target, and FN represents the nonrecognized target.

In order to test the real-time performance of the model, the detection time of each image is measured by recording the experimental process. The images which are selected from the image data set file are used as testing dataset, and the arithmetic average value of each variable is calculated after three times of detection. The probability of error classification can reach 37.962 .

\section{Conclusion}

Pest control is a concern in every region. With the development of science and technology, pest control has a basic policy, but pest control has no particularly obvious effect. In the event of an emergency, Government resources are not sufficient to eliminate pests in their entirety.

In September 2019, a group of wild wasps was found on Vancouver Island, British Columbia, Canada (also known as Asian bumblebees). Although the beehive was soon destroyed, there have been several sightings of the pest since then in neighboring Washington state. Because of the 
potential serious impact on the local bees, as well as causing panic among the masses, the existence of wild wasps caused great anxiety.

In order to deal with the crisis of wild hornet quickly and alleviate the anxiety of the masses, four models (least square method, grey system, convolution neural network, and SIR) were established to analyze the diffusion area and quantity growth trend of wild hornet. It is hoped that by predicting the range of activities and the number of wild wasps, the government can eliminate wild wasps within limited resources.

From 2019 to 2020, the number of wild wasps increased sharply and spread around. By using the least square method and the grey system GM $(1,1)$, we have established the model of the propagation of the wild hornet over time and analyzed its accuracy, but the accuracy is poor because of the influence of other factors such as climate, human factors, and so on.

Because of the low accuracy, in order to increase the reliability of the data, we calculate the sum of the distance from each point to the fitting curve according to the principle of minimum square sum of deviation of the least square method; Otsu algorithm in the least square method is used to fit the data.

Among them, it can be found that the fitting curve of the least square algorithm is very close to the curve of the classical Otsu algorithm, so we can predict the distribution position of the wild hornet by the least square algorithm, combined with the image recognition technology. We can find the location more accurately.

For image recognition technology, we use convolution neural network to process image recognition according to the provided image files. With the increase of network depth, the accuracy rate reaches a bottleneck, thus realizing the possibility of predicting error classification.

We also use the SIR infectious disease model to compare the spread of wild wasps to the spread of infectious diseases. SIR model will give priority to the most likely positive eyewitness reports. In order to ensure the timeliness and accuracy of the model, the model must be updated by changing parameter values or adding parameters according to local conditions. In this way, we can save resources and eliminate wild wasps in a short period of time to ease the anxiety of the masses.

Sometimes, we need to announce the elimination of wild wasps to the masses. In order to facilitate the publication of data to the masses, we have added the Washington state government to control the wild wasps by establishing an optimized SIR infectious disease model. By analyzing the number of infected $I$ (i.e., confirmed as wild wasps) after 250 days, we can prove to the masses that the Washington state has eliminated the pest.

\section{Data Availability}

The data used to support the findings of this study are available from the corresponding author upon request.

\section{Conflicts of Interest}

The authors declare that there are no conflicts of interest regarding the publication of this paper.

\section{Acknowledgments}

This study was funded by Project of Industry and School and Research Institutions in Jiangsu Province of China under Grant no. BY2020626.

\section{References}

[1] T. Fujimoto, Y. Aruka, S. Sechiyama et al., "Sociophysics approach to epidemics," Evolutionary Economics and Social Complexity Science, Springer, Berlin, Germany, 2021.

[2] H. Ito, "Input-to-state stability and Lyapunov functions with explicit domains for SIR model of infectious diseases," American Institute of Mathematical Sciences, vol. 26, no. 9, pp. 5171-5196, 2020.

[3] M. Turkyilmazoglu, "Explicit formulae for the peak time of an epidemic from the SIR model," Physica D Nonlinear Phenomena, vol. 422, Article ID 132902, 2021.

[4] S. Shoukui and X. Sun, Mathematical Modeling Algorithm and Application, National Defense Industry Press, Beijing, China, 2012.

[5] W. Pan, X. Xu, H. Ming, and C. K. Chang, "Clustering mashups by integrating structural and semantic similarities using fuzzy AHP," International Journal of Web Services Research, vol. 18, no. 4, pp. 34-57, 2021.

[6] J. Xu, "Otsu fast image segmentation method based on least square fitting," Journal of Changzhou University (Natural Science Edition), vol. 33, no. 1, pp. 70-76, 2010.

[7] X. Kong, L. Wang, Y. Feng et al., Application and Prospect of Grey Prediction GM $(1,1)$ Model, Qilu University of Technology, Jinan, China, 2018.

[8] G. Dou, R. Liu, and C. Fan, "Identification of examination room misconduct based on convolutional neural network," China Examination, vol. 2021, no. 2, pp. 56-62 + 94, 2021.

[9] S. Li and W. Xie, "Neural network toolbox and application of MATLAB language," Journal of Higher Correspondence Science, vol. 20, no. 1, pp. 43-46, 2007.

[10] J. Luo, P. Feng, A. Haridan et al., MATLAB7.0 Application in Image Processing, Mechanical Industry Press, South Norwalk, CT, USA, 2005.

[11] X. He, Y. Zhou, J. Wang, H. Zhou et al., MATLAB $\times$ Image Processing, Beijing People's Post and Telecommunications Press, Beijing, China, 2006.

[12] X. Lian, Z. Liu, L. Zhang, Y. Han, and G. Shi, "A method for image recognition of retinopathy based on deep learning," Computer Applications and Software, vol. 38, no. 1, pp. 179185, 2021.

[13] D. S. Jayas, J. Paliwal, and N. S. Visen, "Multi-layer neural networks for image analysis of agricultural products," Journal of Agricultural Engineering Research, vol. 77, no. 2, pp. 119$128,2000$.

[14] Four Dimensional Technology, X. Hu, and H. Zhao, Visual CMATLAB Image Processing and Recognition--Practical Case Selection, Beijing: People's Post and Telecommunications Press, Beijing, China, 2004.

[15] Yu Jin, W. Wang, and S. Xiao, "An SIRS model with a nonlinear incidence rate," Chaos, Solitons and Fractals, vol. 34, no. 5, 2006. 